\title{
CATALOGUE OF THE ROZHEN OBSERVATORY SCHMIDT TELESCOPE
}

\author{
A. MUTAFOV, P. ILCHEVA, M. KUSHEVA, M. MICHAILOV, Z. BORISOV and \\ N. LAZAROV \\ Sofia University, Faculty of Physics, Chair of Astronomy \\ James Boucher Blvd. No. 5 \\ BG-1126 Sofia, Bulgaria
}

\section{Introduction}

We present the results from the analysis of the machine-readable version of the log book for the $50 / 70 \mathrm{~cm}$ Schmidt telescope of the National Astronomical Observatory (NAO) Rozhen of the Bulgarian Academy of Sciences. The telescope began operating in 1979, after it was repaired and transferred from the Potsdam Observatory where it had operated since 1952.

For the period from 1979 to April 1993, 6536 plates were obtained; the total effective observational time (EOT) was 2941 hours. We have 2573 plates (1403 hours) with a field of 16.4 sq. deg and 3963 plates (1538 hours) with a field of 25.4 sq. deg. Of these 208 plates (97 hours) are with the 3.5 objective prism, and 334 plates (35 hours) were obtained for technical use (focusing, calibrating etc.). The actual number of plates is 6604 but because of missing data we rejected 68 plates during the analysis.

The telescope is $1750 \mathrm{~m}$ above the sea level and has the parameters given in Golev et al. (1982). Photometric research has shown a good reproduction of the UBVR system (Tsvetkov et al. 1987). Description of the computer-readable version of the catalogue is given in Mutafov et al. (1993).

\section{The Analysis of the Catalogue}

The main part of the observations have been made for specific observational programs, cf. Tsvetkov (1984) and Fig. 1, as follows:

1) investigation of non-stable and flare stars in stellar aggregates;

2) photometric investigation of stellar clusters;

3) observations of small planets in the Solar system;

4) investigation of galaxies;

5) galaxy clusters, etc.

The analysis of the catalog is based on observational material covering the last 14 years. The analysis gives information about the effectivity of the telescope during the whole period of operation. 


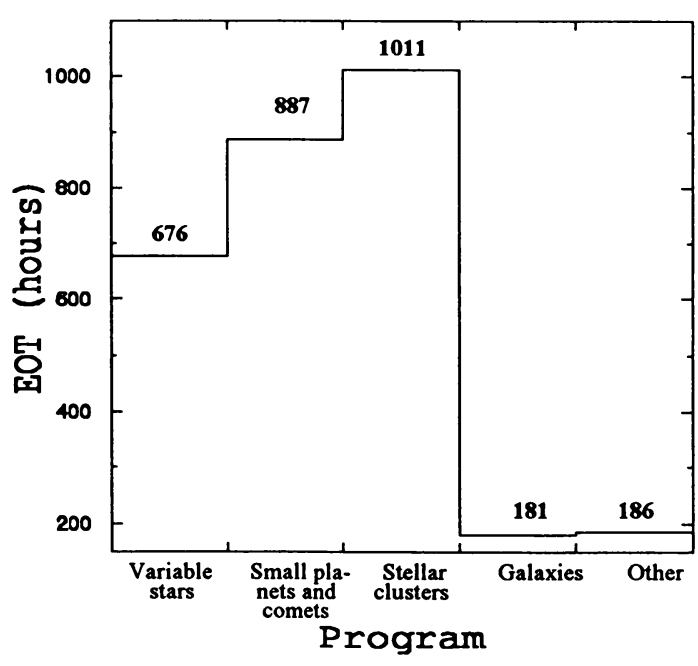

Figure 1. Distribution of the observational programmes by EOT.

Figure 2 shows the distribution of coordinates of plate centres by equatorial coordinates over the sky. It is noticeable that observations are highly concentrated in and around the galactic plane. ORWO plates used during analyzed period total 5710 (2595 hours EOT), and Kodak plates total 794 (343 hours EOT). The most used emulsion is ORWO ZU 21 (5561 plates, 2501 hours EOT).

Table 1 represents number of plates and EOT in each of Johnson and Morgan colours. The higher numbers of plates in $\mathbf{U}$ and $\mathbf{p g}$ are due to patrol observations and the fewer plates in $\mathbf{R}$ and $I$ are due to inappropriate technical parameters of the telescope.

Table 1. Number of plates and effective observational time, by colour

\begin{tabular}{lrrrrrrrl}
\hline Colours & U & \multicolumn{1}{c}{ B } & \multicolumn{1}{c}{ V } & \multicolumn{1}{c}{ R } & I & pg & Other & Total \\
\hline \hline No. plates & 1086 & 1577 & 145 & 191 & 15 & 3129 & 393 & 6536 \\
EOT (hours) & 891 & 661 & 62 & 79 & 11 & 1057 & 180 & 2941 \\
\hline
\end{tabular}

\section{Acknowledgements}

We wish to thank Dr. M. Tsvetkov for the efforts and assistance in accomplishing this work. We are grateful to E. Semkov for the help during the work on the log book of the telescope. We thank P. Ivanov and P. Delchev from Computer Center of Bulgarian Academy of Science, $M$. Matev from Sofia University, and our colleagues from the NAO Rozhen for providing the necessary hardware. 


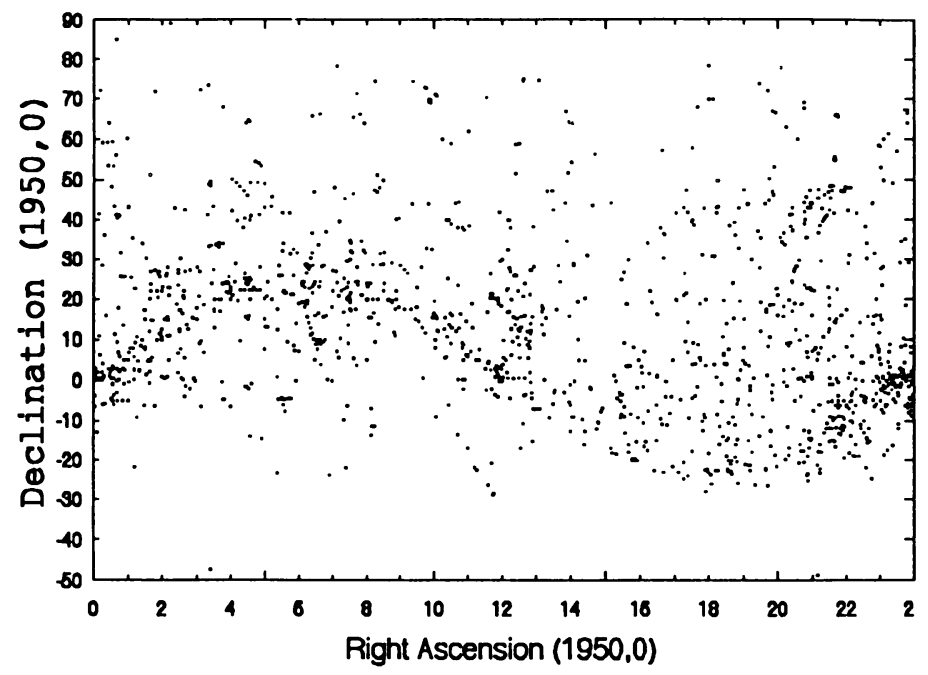

Figure 2. The distribution of the objects by equatorial coordinates.

\section{References}

Golev, V., Tsvetkov, M. and Vitrichenko, E., 1982. Comptes rendus de l'Academie bulgare des Sciences, 35, No. 6, 729.

Mihailov, M.E. and Borisov, Z., 1993. IAU WGWFI Newsletter No. 3, p. 77.

Mutafov, A., Ilcheva, P., Kusheva, M., Mihailov, M.E., Borisov, Z. and Lazarov, N., 1993. IAU WGWFI Newsletter No. 3, p. 83.

Tsvetkov, M., 1984. 'Astronomy with Schmidt-type Telescopes', Proc. of IAU Colloquium No. 78, ed. M. Capaccioli, p. 207.

Tsvetkov, M., Georgiev, T., Bilkina, B., Tsvetkova, A. and Semkov, E., 1987. Comptes rendus de l'Academie bulgare des Sciences, 40, No. 5, 9. 